\title{
Stability of Intelligent Transportation Network Dynamics: A Daily Path Flow Adjustment considering Travel Time Differentiation
}

\author{
Ming-Chorng Hwang, ${ }^{1}$ Hsun-Jung Cho, ${ }^{2}$ and You-Heng Huang ${ }^{2}$ \\ ${ }^{1}$ Microelectronics and Information Systems Research Center, National Chiao Tung University, 1001 University Road, \\ Hsinchu 300, Taiwan \\ ${ }^{2}$ Department of Transportation and Logistics Management, National Chiao Tung University, 1001 University Road, \\ Hsinchu 300, Taiwan \\ Correspondence should be addressed to Ming-Chorng Hwang; mchwang1966@nctu.edu.tw
}

Received 7 October 2014; Revised 13 February 2015; Accepted 15 February 2015

Academic Editor: Yan-Jun Liu

Copyright (C) 2015 Ming-Chorng Hwang et al. This is an open access article distributed under the Creative Commons Attribution License, which permits unrestricted use, distribution, and reproduction in any medium, provided the original work is properly cited.

A theoretic formulation on how traffic time information distributed by ITS operations influences the trajectory of network flows is presented in this paper. The interactions between users and ITS operator are decomposed into three parts: (i) travel time induced path flow dynamics (PFDTT); (ii) demand induced path flow dynamics (PFDD); and (iii) predicted travel time dynamics for an origin-destination (OD) pair (PTTDOD). PFDTT describes the collective results of user's daily route selection by pairwise comparison of path travel time provided by ITS services. The other two components, PTTDOD and PFDD, are concentrated on the evolutions of system variables which are predicted and observed, respectively, by ITS operators to act as a benchmark in guiding the target system towards an expected status faster. In addition to the delivered modelings, the stability theorem of the equilibrium solution in the sense of Lyapunov stability is also provided. A Lyapunov function is developed and employed to the proof of stability theorem to show the asymptotic behavior of the aimed system. The information of network flow dynamics plays a key role in traffic control policy-making. The evaluation of ITS-based strategies will not be reasonable without a well-established modeling of network flow evolutions.

\section{Introduction}

In general, a realizable adjustment process of network flow via intelligent transportation systems (ITS) services must include mechanisms for alleviating congested facilities. Recent developments in communication and information technologies offer opportunities for distributing traffic information to road users and thereby improving system performance. To evaluate ITS-related strategies, it is essential to design a mechanism being able to estimate the interaction between traffic information provision and the corresponding feedbacks of users. Nonequilibrium approach of dynamic traffic assignment (DTA) provides opportunities to relax the restrictive equilibrium assumption and to model phenomena of evolving disequilibria. This inherent property makes it possible to incorporate ITS-based learning mechanism with evolutionary system behaviour more flexibly. Experimental evidence of user decision-making behaviour that involved 100 commuters over a 24 -day period had been performed [ 1 , 2]. The results of these serial studies revealed that route choice of commuters had indicated that the learning and adaptive process for route choice may take weeks. Recently, a similar experimental study is referred to [3]. However, simulationbased method cannot provide a satisfied theoretical analysis of system state to sustain issues of existence, uniqueness, and stability [4]. In the context of day-to-day network dynamics, especially in presence of information, it is likely that users' perception of network performance will vary depending on their experience, accessibility to ITS, personal attributes, and so forth. By allowing the weights to vary across individuals, an extension was used in the experimental study $[5,6]$. Acting as an essential role to have the consequent results 
of route choice, various perception models of travel time were reported [7]. By considering different route choice, dynamical system models were reported and studied, where a tatonnement process in microeconomic theory for modelling the transition of disequilibria from one state to another was examined [8], where a dynamical system composed of nonlinear ordinary differential equations successfully conquering issues of existence and uniqueness was investigated [9-11]. The adaptive-learning behaviour of commuters was actually observed in historical studies. Nonequilibrium approaches are better than equilibrium methods [12-14] with respect to modelling the temporal evolutions between information provision and the consequent responses of users. Analytical techniques own much richness in theoretic issues of system behaviours. However, a definite treatment of how flows shift among routes is extremely necessary for evolutionary models. It will be an interesting study for us to model and study dayto-day flow evolution of an advanced ITS vehicular network.

The focus of this study is to provide a formulation to describe the daily network flow dynamics interacting with the travel cost information distributed by intelligent transportation systems. Even the system behavior/characteristics discussed is assumed to be deterministic, one-day time-lag, and without perception of uncertainty, it still provides a simple but essential formulation to illustrate system dynamics which a traffic control cannot work without. As already wellknown, there are a lot of mature studies in automatic control which is basically the same problem nature as dynamic control in traffic engineering, for example, the $H_{\infty}$ fuzzy control of uncertain active suspension systems [15], the output feedback $H_{\infty}$ control for a class of active quarter-car suspension systems with control delay [16], and the faulttolerant control of Markovian jump stochastic systems [17]. And, there are many studies involving identification and adaptive control of nonlinear dynamical systems using neural networks [18-23]. Similar methodology had been applied in traffic control, for example, [24-26]. It can be found that all these automatic control based approaches cannot work without an essential component to describe system dynamics which is the focus of this study.

This paper attempts to formulate how traffic information distributed by ITS operations influences the temporal trajectory of network flows in a theoretical viewpoint. Based on two behavioral assumptions, minimal-travel-time seeking and adaptive-learning process, the interactions between road users and ITS operator are decomposed into three parts: travel time induced path flow dynamics $\left(\mathrm{PFD}_{\mathrm{TT}}\right)$, demand induced path flow dynamics $\left(\mathrm{PFD}_{\mathrm{D}}\right)$, and predicted travel time dynamics for an origin-destination (OD) pair $\left(\mathrm{PTTD}_{\mathrm{OD}}\right) \cdot \mathrm{PFD}_{\mathrm{TT}}$ describes the collective results of user's daily travel decision by swap to less congested path with path travel time information provided by ITS services. The other two components, $\mathrm{PTTD}_{\mathrm{OD}}$ and $\mathrm{PFD}_{\mathrm{D}}$, are concentrated on the evolutionary behavior of system variables predicted by ITS operators to act as a benchmark in guiding whole systems towards an expected and preferable status based on the observed situations by ITS surveillance system. In addition to the delivered modeling, the stability theorem of the equilibrium solution in the sense of Lyapunov stability is developed and a Lyapunov function is provided and employed to the proof of stability theorem to show the asymptotic behavior of the whole system theoretically. This paper is organized as follows. In Section 2, the proposed model is fully described. Issues of stability are prepared in Section 3. The conclusions of this study are given in Section 4 .

\section{Modelling Network Dynamics}

2.1. Assumptions and General Structure of Dynamics. The main assumptions are: (i) the minimal-travel-time seeking; and (ii) the adaptive-learning behaviour of daily commuters with previous experience and information, together with full travel time information distributed by ITS to determine the shifting preference of path flow. A structure of stimulusresponse relationship (SR) is now given by

$$
\text { response }\left(t+T_{L}\right)=\text { sensivity } \times \text { stimulus }(t),
$$

which is interpreted as a laggard response $\left(T_{L}\right.$ lag) in proportion to the magnitude of stimulus at time $t$. A wellknown application of SR in traffic analysis is the car-following model [27]. The car-following behaviour is formulated as the responsive actions (acceleration or deceleration of the following car) resulting from the stimulus, the perceived velocity difference between the leading vehicle and the following one. To deal with the interaction between information provision and the follow-up travel decisions in the SR-like structure, three assumptions are established: (i) all responses and stimuli discussed hereinafter are macroscopic and divided into two clusters, users and operator; (ii) the response is defined as the time change rate of system variable with oneday lag and denoted as a function of stimulus and sensitivity; and (iii) the stimulus is specified as the difference between experienced (or observed) status and expected (or predicted) status for a system variable. The basic structure of network dynamics is

$$
\begin{gathered}
\dot{R}_{i}^{t} \equiv F_{i}\left(S e_{i}, S t_{i}^{t}\right), \\
S t_{i}^{t} \equiv \widetilde{x}_{i}^{t}-\bar{x}_{i}^{t},
\end{gathered}
$$

where $\dot{R}_{i}^{t}$ is the time change rate (time derivative) of system variable $i$ at day $t ; S t_{i}^{t}$ is the stimulus $i$ at day $t ; S e_{i}$ is the sensitivity of system variable $i$ due to $S t_{i}^{t} ; F_{i}\left(S e_{i}, S t_{i}^{t}\right)$ is a function to transform stimulus into response; $\tilde{x}_{i}^{t}$ is the experienced (or observed) status of system variables $i$ at day $t$ and $\bar{x}_{i}^{t}$ is the expected (or predicted) status of system variables $i$ at day $t ; i$ is the index of system variable referred to the concern of either road users or ITS operators; and $\dot{y}$ denotes the time derivative of $y$.

2.2. User Dynamics. The first user dynamics are the travel time induced path flow dynamics, $\mathrm{PFD}_{\mathrm{TT}}$. By similar proposition [28], the intention of developing $\mathrm{PFD}_{\mathrm{TT}}$ is straightforward that if users wish to improve travel time tomorrow, they might select a faster path than today. To accomplish this end, minimal-travel-time seeking and travel time information provided by ITS operator are necessarily assumed. To fit SR 
structure, we assume that flow shift is related to travel time difference between two paths:

$$
\begin{array}{r}
\dot{h}_{p, c}^{w} \equiv \sum_{j \in P_{w}} \alpha_{w}^{c}\left(c_{j}^{w}-c_{p}^{w}\right) \cong \alpha_{w}^{c} \bar{P}_{w}\left(\widetilde{c}^{w}-c_{p}^{w}\right), \\
\forall t, p \in P_{w}, \quad w \in W,
\end{array}
$$

where $\dot{h}_{p, c}^{w}$ is the cost-induced path flow dynamics of path $p \in$ $P_{w} ; P_{w}$ is the path set of OD pair $w$ containing $\bar{P}_{w}$ paths; $W$ is the set of OD pairs containing $\bar{W}$ OD pairs; $\alpha_{w}^{c}$ is the positive parameter to reflect the sensitivity of path flow dynamics with respect to the cost difference among the paths connecting OD pair $w$; $c_{p}^{w}$ is the cost of path $p \in P_{w}, c_{p}^{w}=\sum_{a \in A} \delta_{a}^{p} c_{a}\left(f_{a}\right)$, $\delta_{a}^{p}=1$ if $a \in$ path $p$, zero otherwise, $A$ is the arc set, and $f_{a}$ is the flow on link $a$; and $\widetilde{c}^{w}$ is the average of all path costs of OD pair $w=1^{\prime} c^{w} / \bar{P}_{w}$, where $c^{w}=\left[\begin{array}{c}c_{1}^{w} \\ \vdots \\ c_{\bar{P}_{w}}^{w}\end{array}\right]$.

The second term of (3) implies the collective effects of pairwise comparison in a way that gave two paths denoted by path $j$ and path $p$; if the cost of path $j$ is greater (less) than path $p$ at day $t$ then path flow will shift from path $j$ (path $p$ ) to path $p$ (path $j$ ) at day $t+1$. Therefore, the term, $\alpha_{w}^{c}\left(c_{j}^{w}-c_{p}^{w}\right)$, in $\dot{h}_{p, c}^{w}$ is positive (negative). It also results in that the term, $\alpha_{w}^{c}\left(c_{p}^{w}-c_{j}^{w}\right)$, in $\dot{h}_{j, c}^{w}$ is negative (positive) at the same time. The third term in (3) is an approximation of the collective effects of pairwise comparison if we assumed that the average of path costs of OD pair $w, \widetilde{c}^{w}$, is approximated by the arithmetic mean of all path costs. Then, we have the following result:

$$
\begin{aligned}
\dot{h}_{p, c}^{w} & \equiv \sum_{j \in P_{w}} \alpha_{w}^{c}\left(c_{j}^{w}-c_{p}^{w}\right)=\left(\alpha_{w}^{c} \sum_{j \in P_{w}} c_{j}^{w}\right)-\alpha_{w}^{c} \bar{P}_{w} c_{p}^{w} \\
& =\left(\alpha_{w}^{c} \bar{P}_{w} \widetilde{c}^{w}\right)-\alpha_{w}^{c} \bar{P}_{w} c_{p}^{w}=\alpha_{w}^{c} \bar{P}_{w}\left(\widetilde{c}^{w}-c_{p}^{w}\right) .
\end{aligned}
$$

It implies immediately that the travel time induced path flow dynamics $\left(\mathrm{PFD}_{\mathrm{TT}}\right)$ for a path $p$ due to the collective effects of pairwise comparison can be approximated in the sense of direct comparison between the average of path costs and the travel cost of path $p$. Shortly speaking, path flow will decrease (increase) next day if the travel cost of a path is greater (less) than the arithmetic mean of all path costs today.

The link cost function $c_{a}\left(f_{a}\right)$ is assumed smooth and strict monotone with respect to the link flow itself. Accordingly, $c_{p}^{w}$ and $\widetilde{c}^{w}$, two functions composed of the set of link cost function, are also smooth and strict monotone with respect to the full set of link flows. And the parameter $\alpha_{w}^{c}$ is assumed small enough to conserve the nonnegativity of path flow. Then we express (3) for all paths of OD pair $w$ in vector form as

$$
\dot{h}_{w, c}=\left[\begin{array}{c}
\dot{h}_{1, c}^{w} \\
\vdots \\
\dot{h}_{\bar{P}_{w}, c}^{w}
\end{array}\right] \cong \alpha_{w}^{c} \bar{P}_{w}\left[\begin{array}{c}
\widetilde{c}^{w}-c_{1}^{w} \\
\vdots \\
\widetilde{c}^{w}-c_{\bar{P}_{w}}^{w}
\end{array}\right]=A_{c}\left(\widetilde{C}^{w}-c^{w}\right) \text {, }
$$

where

$$
\widetilde{C}^{w}=\left[\begin{array}{c}
\widetilde{c}^{w} \\
\vdots \\
\widetilde{c}^{w}
\end{array}\right]_{\bar{P}_{w} \times 1}, \quad A_{w}^{c}=\left[\begin{array}{ccc}
\alpha_{w}^{c} \bar{P}_{w} & 0 & 0 \\
0 & \ddots & 0 \\
0 & 0 & \alpha_{w}^{c} \bar{P}_{w}
\end{array}\right]_{\bar{P}_{w} \times \bar{P}_{w}} .
$$

We can further express (5) for full set of paths in vector form as

$$
\dot{h}_{c} \cong A_{c}\left(\Gamma \widetilde{C}-\Delta^{\prime} C_{a}(\Delta h)\right),
$$

where $A_{c}=\left[\begin{array}{ccc}A_{1}^{c} & 0 & 0 \\ 0 & \ddots & 0 \\ 0 & 0 & A_{\bar{W}}^{c}\end{array}\right] ; C_{a}(\Delta h)$ is full set of link costs; $\widetilde{C}$ is the full set of the average of path cost for OD pair set $W ; h$ is the full set of path flows; $\Gamma$ and $\Delta$ are path-OD pair incidence matrix and link-path incidence matrix, respectively; and $x^{\prime}$ denotes the transpose of $x$.

The stimulus of $\mathrm{PFD}_{\mathrm{TT}}$ is the collective effects of travel time difference prevailing on OD pair $w$. This is so-called the travel time induced path flow dynamics. For the second expression in (3), similarly again the stimulus of $\mathrm{PFD}_{\mathrm{TT}}$ is reinterpreted as the difference between the average experienced travel time and the path travel time of interest. With the provided full information of travel time, $\mathrm{PFD}_{\mathrm{TT}}$ constitutes the whole process of path flow swapping and then determines the evolution of network flow dynamics.

2.3. ITS Operator Dynamics. To forward network traffics to a stable status is the major intent of the ITS operator. In this subsection, ITS operator dynamics are presented to fulfill this end. The results of user dynamics are performed on the road network and observed by ITS devices. With similar adaptive and learning process mentioned in previous section, ITS operators utilize the detected information to compare and update some predictable system variables. The first one is demand induced path flow dynamics, $\mathrm{PFD}_{\mathrm{D}}$, which is denoted by

$$
\dot{h}_{p, d}^{w} \equiv \alpha_{p, w}^{d}\left(d^{w}-h^{w}\right), \quad \forall t, p \in P_{w}, w \in W,
$$

where $\dot{h}_{p, d}^{w}$ is the demand induced path flow dynamics of path $p \in P_{w} ; \alpha_{p, w}^{d}$ is the positive parameter to reflect the sensitivity of path flow dynamics with respect to the difference between the predicted demand and the sum of observed path flows on OD pair $w$, moreover $\alpha_{p, w}^{d}$ is assumed to be weighted over the path costs of OD pair $w$; $d^{w}$ is the fixed demand of OD pair $w, \forall w \in W ; h^{w}$ is the sum of path flows $\forall w \in W$.

The parameter $\alpha_{p, w}^{d}$ is also assumed small enough to conserve the nonnegativity of path flow. Then we express (8) for all paths of OD pair $w$ in vector form as

$$
\dot{h}_{w, d}=\left[\begin{array}{c}
\dot{h}_{1, d}^{w} \\
\vdots \\
\dot{h}_{\bar{P}_{w}, d}^{w}
\end{array}\right]=A_{w}^{d}\left[\begin{array}{c}
d^{w}-h^{w} \\
\vdots \\
d^{w}-h^{w}
\end{array}\right]
$$


where

$$
A_{w}^{d}=\left[\begin{array}{ccc}
\alpha_{1, w}^{d} & 0 & 0 \\
0 & \ddots & 0 \\
0 & 0 & \alpha_{\bar{P}_{w}, w}^{d}
\end{array}\right]_{\bar{P}_{w} \times \bar{P}_{w}}
$$

We can further express (9) for full set of paths in vector form as

$$
\dot{h}_{d}=A_{d} \Gamma\left(D-\Gamma^{\prime} h\right),
$$

where $A_{d}=\left[\begin{array}{ccc}A_{1}^{d} & 0 & 0 \\ 0 & \ddots & 0 \\ 0 & 0 & A_{\bar{W}}^{d}\end{array}\right]$ and $D$ is the full set of the predicted demands.

The stimulus described in (8) is the difference between path flow sum and demand for an OD pair $w \in W$. The path flow sum is "observed and calculated" by ITS operator. The demand of an OD pair $w$, assumed fixed in this study, is interpreted as a predicted/expected status of network by ITS operator. This dynamic provides the adjustment from the observed path flow sum to the expected demand which can only be achieved by ITS operator. By assumed constant OD demands, this study attempts to concentrate on system dynamics caused only by the effect of travel time provision.

The difference between path flow sum and demand simultaneously generates an effect on predicted travel time for an OD pair, PTTD $\mathrm{OD}$. This effect forms the second ITS operator dynamics written as

$$
\dot{\tilde{c}}_{w} \equiv \beta_{w}\left(d^{w}-h^{w}\right), \quad \forall t, w \in W,
$$

where $\dot{\tilde{c}}_{w}$ is the predicted cost dynamics of OD pair $w \in W$; $\beta_{w}$ is the positive parameter to reflect the sensitivity of predicted cost dynamics with respect to the difference between the predicted demand and the sum of observed path flows on OD pair $w$.

The parameter $\beta_{w}$ is also assumed small enough to conserve the nonnegativity of predicted cost.

Then we express (12) for all OD pairs in vector form as

$$
\dot{\widetilde{C}}=B\left(D-\Gamma^{\prime} h\right),
$$

where $B=\left[\begin{array}{ccc}\beta_{1} & 0 & 0 \\ 0 & \ddots & 0 \\ 0 & 0 & \beta_{\bar{W}}\end{array}\right]$ and $\widetilde{C}$ is the predicted costs of full OD pairs set $W$.

To sum up, we combine (7), (11), and (13) as

$$
\left(\begin{array}{c}
\dot{h} \\
\dot{\widetilde{C}}
\end{array}\right)=\left[\begin{array}{cc}
A_{c} \mid A_{d} & 0 \\
0 & B
\end{array}\right]\left(\left[\begin{array}{c}
\left.\frac{\Gamma \widetilde{C}-\Delta^{\prime} c_{a}(\Delta h)}{\Gamma\left(D-\Gamma^{\prime} h\right)}\right] \\
D-\Gamma^{\prime} h
\end{array}\right) .\right.
$$

\section{Stability Analysis}

3.1. Analysis of Steady State. This section describes the steady state of proposed network dynamics which meets Wardrop's user equilibrium [29]. We first collect the formulation together and proceed to the advantageous results. The dynamics are shown in (3), (8), and (12).

It is obvious that the steady state of (12) gives the fact that $d^{w}=h^{w}, \forall w \in W$. It converges paths flow to demand of OD pair. For the steady state of path flow dynamics which is the sum (net effects) of (8) and (3), we express

$$
\begin{array}{r}
\dot{h}_{p}^{w}=\dot{h}_{p, c}^{w}+\dot{h}_{p, d}^{w}=\left(\sum_{j \in P_{w}} \alpha_{w}^{c}\left(c_{j}^{w}-c_{p}^{w}\right)\right)+\alpha_{p, w}^{d}\left(d^{w}-h^{w}\right), \\
\forall p \in P_{w}, \quad w \in W .
\end{array}
$$

The steady state of (12) implies $d^{w}=h^{w}, \forall w \in W$, and helps us to focus the case of (15) on $\sum_{j \in P_{w}} \alpha_{w}^{c}\left(c_{j}^{w}-c_{p}^{w}\right)$ which is the sum of cost differences between path $p$ and each path $j \in P_{w}$, respectively. Each term of cost difference in (15) should be zero if the path with minimal (maximal) cost is considered and steady state is reached. Because the amount of each team should be positive (negative) or equal to zero for the path with minimal (maximal) cost, this situation together with their sum being equal to zero (condition of steady state) jointly gives that each term of cost difference in (15) should be zero, namely, $c_{j}^{w}=c_{p}^{w}, \forall j \in P_{w}, j \neq p, w \in W$, if steady state is reached. This result justifies that the steady states of (3), (8), and (12) meet Wardrop's user equilibrium. However, we need to assume that the path flow is positive on steady state for all the path set considered.

3.2. Stability of Equilibrium Solution. In this section, the proposed dynamical system (14) is shown to be asymptotically stable in the sense of Lyapunov. The definition of Lyapunov function and theorem of stability are collected first as in the following [30].

Definition 1. Let $\bar{v}$ be a steady state of a dynamical system and $G \in C^{1}(E)$. A function $L: E \rightarrow R$ is called a strict Lyapunov function for $\bar{v}$ if the following conditions are satisfied:

(1) $L(\bar{v})=0$, and $L(v)>0, \forall v \neq \bar{v}$, and $v \in E$;

(2) $\dot{L}(v)<0, \forall v \neq \bar{v}$, and $v \in E$.

Theorem 2. Let $\bar{v}$ be a steady state of $\dot{v}=G(v)$. If there exists a strict Lyapunov function $\forall v \neq \bar{v}, v \in E$, then $\bar{v}$ is asymptotically stable.

Accordingly, the stability theorem of proposed dynamical system is expressed as Theorem 3. Because of the parameter matrix in (14), $\left[\begin{array}{ccc}A_{c} \mid A_{d} & 0 \\ 0 & B\end{array}\right]$, not being a square matrix, it will be difficult to find a Lyapunov function if we prefer to find 
a Lyapunov function in quadratic form. We apply (16) to be an equivalent agent of (14) on the following analysis:

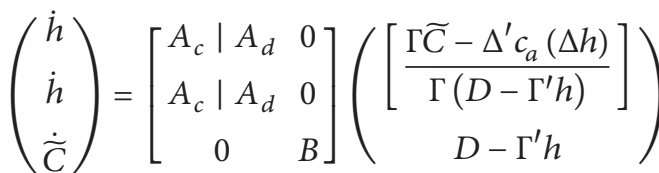

$$
\begin{aligned}
& =\varphi\left(M(s)+\Omega\left(\begin{array}{l}
h \\
h \\
\widetilde{C}
\end{array}\right)\right),
\end{aligned}
$$

where

$$
\begin{gathered}
\varphi=\left[\begin{array}{cc}
A_{c} \mid A_{d} & 0 \\
A_{c} \mid A_{d} & 0 \\
0 & B
\end{array}\right], \quad s \equiv\left(\begin{array}{c}
\Delta h \\
\Delta h \\
\widetilde{C}
\end{array}\right), \\
M(s) \equiv\left(\begin{array}{cc}
-\Delta^{\prime} c_{a}(\Delta h) \\
\Gamma D \\
D
\end{array}\right) \\
\Omega \equiv\left(\begin{array}{ccc}
0 & 0 & \Gamma \\
-\frac{\Gamma \Gamma^{\prime}}{2} & -\frac{\Gamma \Gamma^{\prime}}{2} & 0 \\
-\frac{\Gamma^{\prime}}{2} & -\frac{\Gamma^{\prime}}{2} & 0
\end{array}\right)
\end{gathered}
$$

Theorem 3. Let $\left(\frac{\bar{h}}{\frac{\bar{h}}{\mathrm{C}}}\right)^{\prime} \equiv\left(\bar{h}_{1}, \bar{h}_{2}, \ldots, \bar{h}_{\bar{P}}, \bar{h}_{1}, \bar{h}_{2}, \ldots, \bar{h}_{\bar{P}}, \bar{C}_{1}\right.$, $\left.\bar{C}_{2}, \ldots, \bar{C}_{\bar{W}}\right)$ be a steady state of (16) and $\left(\frac{\bar{h}}{\bar{h}}\right)$ is asymptotically stable.

Proof. Let $L$ be a $C^{1}$ map and

$$
\begin{aligned}
& L\left(\begin{array}{l}
h \\
h \\
\widetilde{C}
\end{array}\right) \\
& \quad \equiv \frac{1}{2}\left(\left(\begin{array}{c}
h \\
h \\
\widetilde{C}
\end{array}\right)-\left(\begin{array}{c}
\bar{h} \\
\bar{h} \\
\bar{C}
\end{array}\right)\right)^{\prime} \psi \widetilde{\varphi}\left(\left(\begin{array}{l}
h \\
h \\
\widetilde{C}
\end{array}\right)-\left(\begin{array}{l}
\bar{h} \\
\bar{h} \\
\bar{C}
\end{array}\right)\right),
\end{aligned}
$$

It is easy to show that $L\left(\begin{array}{c}\bar{h} \\ \bar{h} \\ \bar{C}\end{array}\right)=0$ and $L\left(\begin{array}{c}h \\ h \\ \tilde{C}\end{array}\right)>0, \forall\left(\begin{array}{c}h \\ h \\ \widetilde{C}\end{array}\right) \neq$ $\left(\begin{array}{l}\bar{h} \\ \bar{h}\end{array}\right)$.

Now we proceed to the second condition of Definition 1 and take the derivative of (18):

$$
\dot{L}\left(\begin{array}{c}
h \\
h \\
\widetilde{C}
\end{array}\right)=\left(\left(\begin{array}{c}
h \\
h \\
\widetilde{C}
\end{array}\right)-\left(\begin{array}{c}
\bar{h} \\
\bar{h} \\
\bar{C}
\end{array}\right)\right)^{\prime} \psi \widetilde{\varphi}\left(\begin{array}{c}
\dot{h} \\
\dot{h} \\
\dot{\widetilde{C}}
\end{array}\right)
$$

$$
=-\left(\left(\begin{array}{l}
h \\
h \\
\widetilde{C}
\end{array}\right)-\left(\begin{array}{l}
\bar{h} \\
\bar{h} \\
\bar{C}
\end{array}\right)\right)^{\prime}
$$$$
\cdot \psi \varphi \widetilde{\varphi}\left(M(s)+\Omega\left(\begin{array}{l}
h \\
h \\
\widetilde{C}
\end{array}\right)\right)
$$$$
=\left(\left(\begin{array}{c}
h \\
h \\
\widetilde{C}
\end{array}\right)-\left(\begin{array}{l}
\bar{h} \\
\bar{h} \\
\bar{C}
\end{array}\right)\right)^{\prime} \psi\left(\begin{array}{lll}
I & 0 & 0 \\
I & 0 & 0 \\
0 & 0 & I
\end{array}\right)
$$

$$
\cdot\left(M(s)+\Omega\left(\begin{array}{l}
h \\
h \\
\widetilde{C}
\end{array}\right)\right)
$$$$
=\left(\left(\begin{array}{l}
h \\
h \\
\widetilde{C}
\end{array}\right)-\left(\begin{array}{l}
\bar{h} \\
\bar{h} \\
\bar{C}
\end{array}\right)\right)^{\prime}\left(\begin{array}{ccc}
I & 0 & 0 \\
I & 0 & 0 \\
0 & 0 & 2 I
\end{array}\right)
$$

$$
\begin{aligned}
& \left(M(s)+\Omega\left(\begin{array}{l}
h \\
h \\
\widetilde{C}
\end{array}\right)\right) \\
= & -\left(\left(\begin{array}{l}
h \\
h \\
\widetilde{C}
\end{array}\right)-\left(\begin{array}{l}
\bar{h} \\
\bar{h} \\
\bar{C}
\end{array}\right)\right)^{\prime}\left(\widetilde{M}(s)+\widetilde{\Omega}\left(\begin{array}{l}
h \\
h \\
\widetilde{C}
\end{array}\right)\right),
\end{aligned}
$$

where

$$
\widetilde{\varphi} \equiv\left[\begin{array}{ccc}
\frac{1}{2}\left(\frac{A_{c}^{-1}}{A_{d}^{-1}}\right) & \left(\frac{A_{c}^{-1}}{-A_{d}^{-1}}\right) & 0 \\
0 & 0 & B^{-1}
\end{array}\right], \quad \psi \equiv\left[\begin{array}{lll}
I & 0 & 0 \\
0 & I & 0 \\
0 & 0 & 2 I
\end{array}\right] .
$$

where

$$
\widetilde{M}(s) \equiv\left(\begin{array}{c}
\Delta^{\prime} c_{a}(\Delta h) \\
\Delta^{\prime} c_{a}(\Delta h) \\
-2 D
\end{array}\right), \quad \widetilde{\Omega} \equiv\left(\begin{array}{ccc}
0 & 0 & -\Gamma \\
0 & 0 & -\Gamma \\
\Gamma^{\prime} & \Gamma^{\prime} & 0
\end{array}\right) .
$$


Because $c_{a}(\cdot)$ is a strict monotone function, we have $(s-$ $\bar{s})^{\prime}(\widetilde{M}(s)-\widetilde{M}(\bar{s}))>0$; that is, $(s-\bar{s})^{\prime} \widetilde{M}(s)>(s-\bar{s})^{\prime} \widetilde{M}(\bar{s})$. This implies $\left(\left(\begin{array}{c}h \\ h \\ \widetilde{C}\end{array}\right)-\left(\begin{array}{c}\bar{h} \\ \bar{h} \\ \bar{C}\end{array}\right)\right)^{\prime} \widetilde{M}(s)>\left(\left(\begin{array}{c}h \\ h \\ \widetilde{C}\end{array}\right)-\left(\begin{array}{c}\bar{h} \\ \bar{h} \\ \bar{C}\end{array}\right)\right)^{\prime} \widetilde{M}(\bar{s})$. And by the steady state, we also have $\left(\frac{\bar{h}}{\bar{h}}\right)^{\prime}\left(\widetilde{M}(\bar{s})+\widetilde{\Omega}\left(\begin{array}{c}\bar{h} \\ \overline{\bar{C}}\end{array}\right)\right)=$ 0 and $\left(\begin{array}{c}h \\ h \\ \widetilde{C}\end{array}\right)^{\prime}\left(\widetilde{M}(\bar{s})+\widetilde{\Omega}\left(\begin{array}{c}\bar{h} \\ \bar{h} \\ \bar{C}\end{array}\right)\right) \geq 0$. This jointly implies

$$
\begin{gathered}
\left(\left(\begin{array}{l}
h \\
h \\
\widetilde{C}
\end{array}\right)-\left(\begin{array}{l}
\bar{h} \\
\bar{h} \\
\bar{C}
\end{array}\right)\right)^{\prime} \widetilde{M}(s) \\
>\left(\left(\begin{array}{l}
\bar{h} \\
\bar{h} \\
\bar{C}
\end{array}\right)-\left(\begin{array}{l}
h \\
h \\
\widetilde{C}
\end{array}\right)\right) \widetilde{\Omega}\left(\begin{array}{l}
\bar{h} \\
\bar{h} \\
\bar{C}
\end{array}\right) .
\end{gathered}
$$

$\operatorname{Adding}\left(\left(\begin{array}{l}h \\ h \\ \widetilde{C}\end{array}\right)-\left(\begin{array}{c}\bar{h} \\ \bar{h} \\ \bar{C}\end{array}\right)\right)^{\prime} \widetilde{\Omega}\left(\begin{array}{l}h \\ h \\ \widetilde{C}\end{array}\right)$ to both sides of (22) gives

$$
\begin{gathered}
\left(\left(\begin{array}{l}
h \\
h \\
\widetilde{C}
\end{array}\right)-\left(\begin{array}{l}
\bar{h} \\
\bar{h} \\
\bar{C}
\end{array}\right)\right)^{\prime}\left(\widetilde{M}(s)+\widetilde{\Omega}\left(\begin{array}{l}
h \\
h \\
\widetilde{C}
\end{array}\right)\right) \\
>\left(\left(\begin{array}{l}
\bar{h} \\
\bar{h} \\
\bar{C}
\end{array}\right)-\left(\begin{array}{l}
h \\
h \\
\widetilde{C}
\end{array}\right)\right)^{\prime}\left(\widetilde{\Omega}\left(\begin{array}{l}
\bar{h} \\
\bar{h} \\
\bar{C}
\end{array}\right)-\widetilde{\Omega}\left(\begin{array}{l}
h \\
h \\
\widetilde{C}
\end{array}\right)\right)
\end{gathered}
$$

That is,

$$
\begin{gathered}
\left(\left(\begin{array}{l}
h \\
h \\
\widetilde{C}
\end{array}\right)-\left(\begin{array}{l}
\bar{h} \\
\bar{h} \\
\bar{C}
\end{array}\right)\right)^{\prime}\left(\widetilde{M}(s)+\widetilde{\Omega}\left(\begin{array}{l}
h \\
h \\
\widetilde{C}
\end{array}\right)\right) \\
>\left(\left(\begin{array}{l}
\bar{h} \\
\bar{h} \\
\bar{C}
\end{array}\right)-\left(\begin{array}{l}
h \\
h \\
\widetilde{C}
\end{array}\right)\right)^{\prime} \widetilde{\Omega}\left(\left(\begin{array}{l}
\bar{h} \\
\bar{h} \\
\bar{C}
\end{array}\right)-\left(\begin{array}{l}
h \\
h \\
\widetilde{C}
\end{array}\right)\right)
\end{gathered}
$$

Following some further treatments on the right-hand side of the above equation,

$$
\begin{aligned}
& \left(\begin{array}{c}
\bar{h}-h \\
\bar{h}-h \\
\bar{C}-\widetilde{C}
\end{array}\right)^{\prime}\left(\begin{array}{ccc}
0 & 0 & -\Gamma \\
0 & 0 & -\Gamma \\
\Gamma^{\prime} & \Gamma^{\prime} & 0
\end{array}\right)\left(\begin{array}{c}
\bar{h}-h \\
\bar{h}-h \\
\bar{C}-\widetilde{C}
\end{array}\right) \\
& =\left(\begin{array}{c}
\bar{h}-h \\
\bar{h}-h \\
\bar{C}-\widetilde{C}
\end{array}\right)\left(\begin{array}{c}
-\Gamma(\bar{C}-\widetilde{C}) \\
-\Gamma(\bar{C}-\widetilde{C}) \\
2 \Gamma^{\prime}(\bar{h}-h)
\end{array}\right) \\
& =-2(\bar{h}-h)^{\prime} \Gamma(\widetilde{C}-\bar{C})+2(\bar{C}-\widetilde{C})^{\prime} \Gamma^{\prime}(\bar{h}-h) \\
& =-2(\widetilde{C}-\bar{C})^{\prime} \Gamma^{\prime}(\bar{h}-h)+2(\bar{C}-\widetilde{C})^{\prime} \Gamma^{\prime}(\bar{h}-h)=0 .
\end{aligned}
$$

Now, recall $\dot{L}\left(\begin{array}{c}h \\ h \\ \widetilde{C}\end{array}\right)=-\left(\left(\begin{array}{c}h \\ h \\ \widetilde{C}\end{array}\right)-\left(\begin{array}{c}\bar{h} \\ \bar{h} \\ \bar{C}\end{array}\right)\right)^{\prime}\left(\widetilde{M}(s)+\widetilde{\Omega}\left(\begin{array}{c}h \\ h \\ \widetilde{C}\end{array}\right)\right) ;$ thus apparently we have $\dot{L}\left(\begin{array}{l}h \\ h \\ \widetilde{C}\end{array}\right)<0$.

\section{Conclusions and Future Research}

In this paper, a model has been developed to investigate the effects of traffic information provision on network flow evolutions under scenario of ITS operations. Traffic information provided to users is path travel time to help users perform comparative reasoning in adaptive-learning process. The whole system coupling both user and operator dynamics is organized as three parts: travel time induced path flow dynamics $\left(\mathrm{PFD}_{\mathrm{TT}}\right)$, predicted travel time dynamics for an OD pair $\left(\mathrm{PTTD}_{\mathrm{OD}}\right)$, and demand induced path flow dynamics $\left(\mathrm{PFD}_{\mathrm{D}}\right)$. They were formulated as a nonlinear system of ordinary differential equations. And a Lyapunov function is created to help the proof of stability theorem. In particular, the effect of intershifting of path flow was successfully considered in $\mathrm{PFD}_{\mathrm{TT}}$. In addition to the developed theory, it concurrently provided a way to help decision-making in traffic control. By collecting path flow shifting information, traffic control strategies can then be simulated in transition state but equilibrium state which usually appeared in historical studies. That will be an important factor to make the results of evaluating the effectiveness and efficiency of ITSrelated deployments more reasonable. The proposed model can approximate the path flow dynamics involving pairwise comparison in an alternative way of direct comparison between the average of path costs and the travel cost of a corresponding path. Finally, we provide an agent of origin formulation to find a Lyapunov function in quadratic form that is much easier to derive the advantageous results in the proof of stability of equilibrium. The application of the proposed results will be set up on a situation where ITS services are well equipped. For example, the daily path choice, departure time, and arrival time of commuting trips should be well recorded by ITS technologies. Daily path travel time information could be distributed to users in 
return to help them perform pairwise comparisons of travel cost and then make a decision of route selection for daily commuting based on the latest travel information and his/her last travel experience. ITS center could also collect input data and calibrate the parameters necessary in the model. Further issues on how to forward the system to the desired status faster in terms of strategies of information distribution or traffic control policies based on the proposed system dynamics will be very important to alleviate urban traffic congestion.

\section{Conflict of Interests}

The authors declare that there is no conflict of interests regarding the publication of this paper.

\section{Acknowledgments}

The authors would like to thank the Ministry of Transportation and Communications, Taiwan, and the Ministry of Science and Technology, Taiwan, for financially supporting this study (under Contracts MOTC-STAO-103-02, MOTCSTAO-103-03, and 103-2622-E-009-012, resp.).

\section{References}

[1] G.-L. Chang and T. Junchaya, "A dynamic route assignment model for guided and unguided vehicles with a massively parallel computing architecture," Mathematical and Computer Modelling, vol. 22, no. 4-7, pp. 377-395, 1995.

[2] H. S. Mahmassani, G.-L. Chang, and R. Herman, "Individual decisions and collective effects in simulated traffic system," Transportation Science, vol. 20, no. 4, pp. 258-271, 1986.

[3] J. L. Hartman, "Speical issue on transport infrastructure: a route choice experiment with an efficient toll," Networks and Spatial Economics, vol. 12, no. 2, pp. 205-222, 2012.

[4] S. Peeta and A. K. Ziliaskopoulos, "Foundations of dynamic traffic assignment: the past, the present and the future," Networks and Spatial Economics, vol. 1, no. 3-4, pp. 233-266, 2001.

[5] M. Ben-Akiva, A. de Palma, and I. Kaysi, "Dynamic network models and driver information systems," Transportation Research Part A: General, vol. 25, no. 5, pp. 251-266, 1991.

[6] G.-L. Chang and H. S. Mahmassani, "Travel time prediction and departure time adjustment behavior dynamics in a congested traffic system," Transportation Research Part B, vol. 22, no. 3, pp. 217-232, 1988.

[7] M. Jha, S. Madanat, and S. Peeta, "Perception updating and dayto-day travel choice dynamics in traffic networks with information provision," Transportation Research Part C: Emerging Technologies, vol. 6, no. 3, pp. 189-212, 1998.

[8] T. L. Friesz, D. Bernstein, N. J. Mehta, R. L. Tobin, and S. Ganjalizadeh, "Day-to-day dynamic network disequilibria and idealized traveler information systems," Operations Research, vol. 42, no. 6, pp. 1120-1136, 1994.

[9] H.-J. Cho, M.-C. Hwang, and Y.-T. Chiu, "Quasi user equilibrium and its stability," in Proceedings of the IEEE International Conference on Systems, Man and Cybernetics (SMC '04), pp. 3944-3949, October 2004.
[10] H.-J. Cho and M.-C. Hwang, "Day-to-day vehicular flow dynamics in intelligent transportation network," Mathematical and Computer Modelling, vol. 41, no. 4-5, pp. 501-522, 2005.

[11] H.-J. Cho and M.-C. Hwang, "A stimulus-response model of day-to-day network dynamics," IEEE Transactions on Intelligent Transportation Systems, vol. 6, no. 1, pp. 17-25, 2005.

[12] D. K. Merchant and G. L. Nemhauser, "A model and an algorithm for the dynamic traffic assignment problems," Transportation Science, vol. 12, no. 3, pp. 183-199, 1978.

[13] H. K. Lo and W. Y. Szeto, "A cell-based dynamic traffic assignment model: formulation and properties," Mathematical and Computer Modelling, vol. 35, no. 7-8, pp. 849-865, 2002.

[14] A. H. Shahpar, H. Z. Aashtiani, and A. Babazadeh, "Dynamic penalty function method for the side constrained traffic assignment problem," Applied Mathematics and Computation, vol. 206, no. 1, pp. 332-345, 2008.

[15] H. Li, X. Jing, H.-K. Lam, and P. Shi, "Fuzzy sampled-data control for uncertain vehicle suspension systems," IEEE Transactions on Cybernetics, vol. 44, no. 7, pp. 1111-1126, 2014.

[16] H. Li, X. Jing, and H. R. Karimi, “Output-feedback-based $H_{\infty}$ control for vehicle suspension systems with control delay," IEEE Transactions on Industrial Electronics, vol. 61, no. 1, pp. 436-446, 2014.

[17] H. Li, H. Gao, P. Shi, and X. Zhao, "Fault-tolerant control of Markovian jump stochastic systems via the augmented sliding mode observer approach," Automatica, vol. 50, no. 7, pp. 18251834, 2014.

[18] K. S. Narendra and K. Parthasarathy, "Identification and control of dynamical systems using neural networks," IEEE Transactions on Neural Networks, vol. 1, no. 1, pp. 4-27, 1990.

[19] G. A. Rovithakis and M. A. Christodoulou, "Adaptive control of unknown plants using dynamical neural networks," IEEE Transactions on Systems, Man, and Cybernetics, vol. 24, no. 3, pp. 400-412, 1994.

[20] F. C. Chen and H. K. Khalil, "Adaptive control of a class of nonlinear discrete-time systems using neural networks," IEEE Transactions on Automatic Control, vol. 40, no. 5, pp. 791-801, 1995.

[21] Y.-J. Liu, C. L. P. Chen, G.-X. Wen, and S. Tong, "Adaptive neural output feedback tracking control for a class of uncertain discrete-time nonlinear systems," IEEE Transactions on Neural Networks, vol. 22, no. 7, pp. 1162-1167, 2011.

[22] C. L. P. Chen, Y.-J. Liu, and G.-X. Wen, "Fuzzy neural networkbased adaptive control for a class of uncertain nonlinear stochastic systems," IEEE Transactions on Cybernetics, vol. 44, no. 5, pp. 583-593, 2014.

[23] C. L. P. Chen, G.-X. Wen, Y.-J. Liu, and F.-Y. Wang, "Adaptive consensus control for a class of nonlinear multiagent time-delay systems using neural networks," IEEE Transactions on Neural Networks and Learning Systems, vol. 25, no. 6, pp. 1217-1226, 2014.

[24] M. Papageorgiou, Applications of Automatic Control Concepts to Traffic Flow Modeling and Control, vol. 50 of Lecture Notes in Control and Information Sciences, Springer, Berlin, Germany, 1983.

[25] P. Kachroo and K. Özbay, Feedback Control Theory for Dynamic Traffic Assignment, Springer, London, UK, 1999.

[26] P. Kachroo and K. Özbay, Feedback Ramp Metering in Intelligent Transportation Systems, Kluwer Academic/Plenum Publisher, New York, NY, USA, 2003. 
[27] D. C. Gazis, R. Herman, and R. W. Rothery, "Nonlinear followthe-leader models of traffic flow," Operations Research, vol. 9, pp. 545-567, 1961.

[28] M. J. Smith, "The existence, uniqueness and stability of traffic equilibria," Transportation Research Part B: Methodological, vol. 13, no. 4, pp. 295-304, 1979.

[29] J. G. Wardrop, "Some theoretical aspects of road traffic research," in Proceedings of the Institute of Civil Engineers: Part II, pp. 325-378, 1952.

[30] K. T. Alligood, T. D. Sauer, and J. A. Yorke, Chaos: An Introduction to Dynamical Systems, Springer, New York, NY, USA, 1997. 


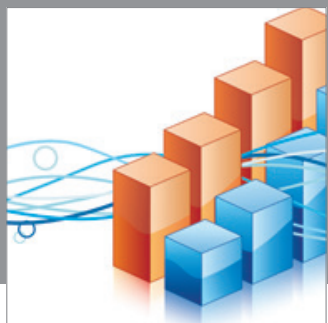

Advances in

Operations Research

mansans

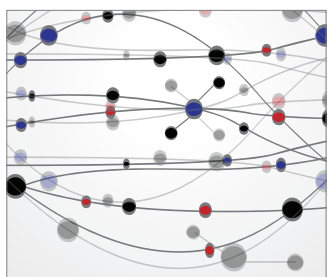

The Scientific World Journal
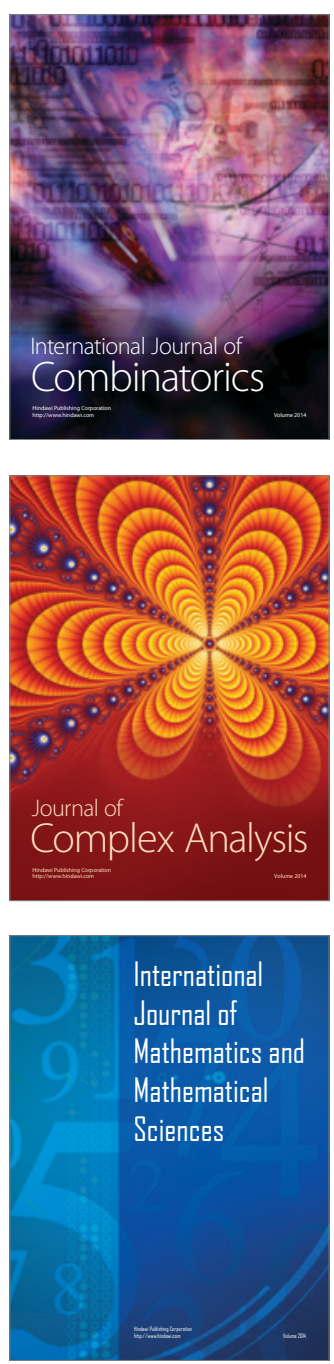
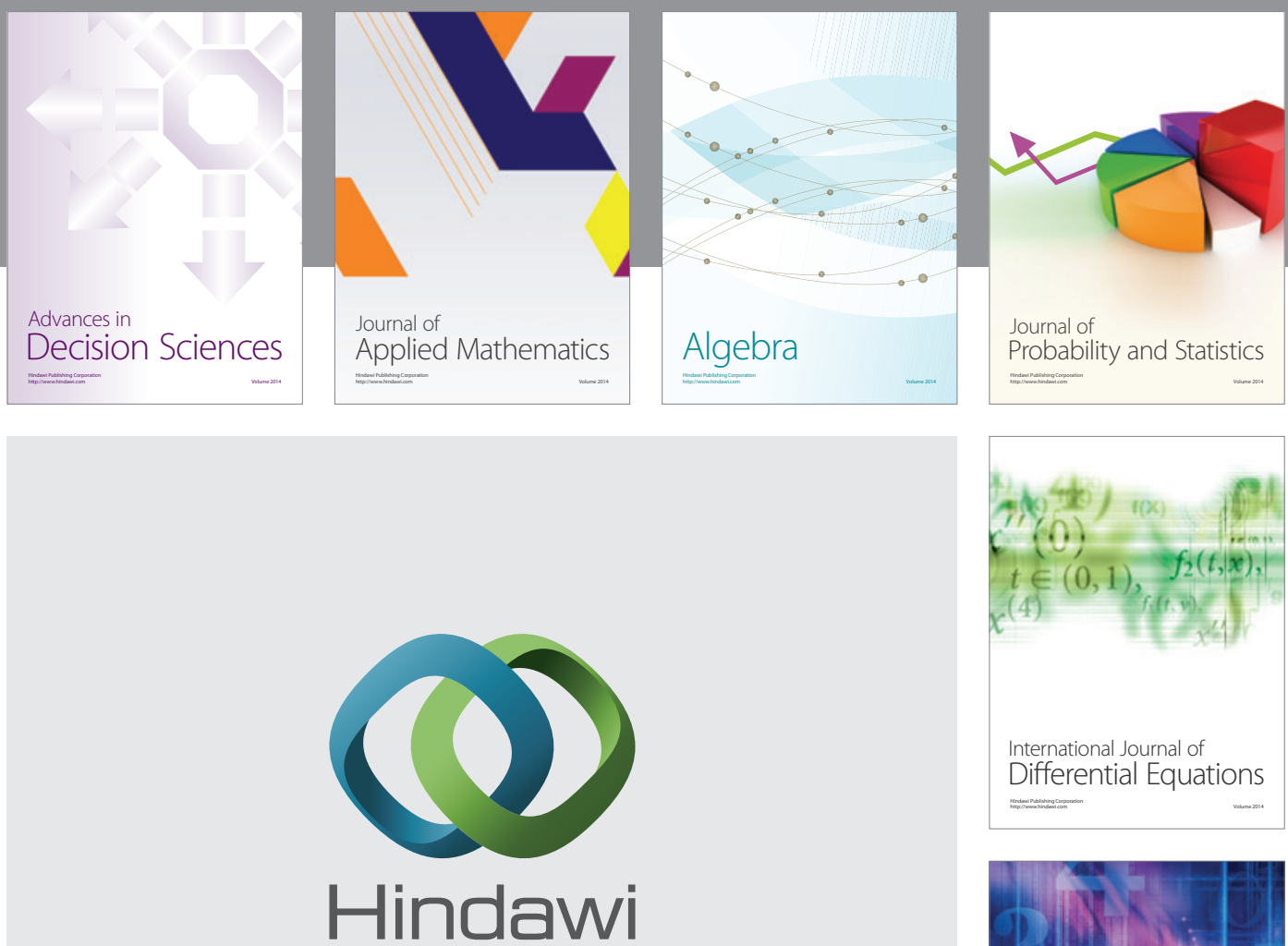

Submit your manuscripts at http://www.hindawi.com
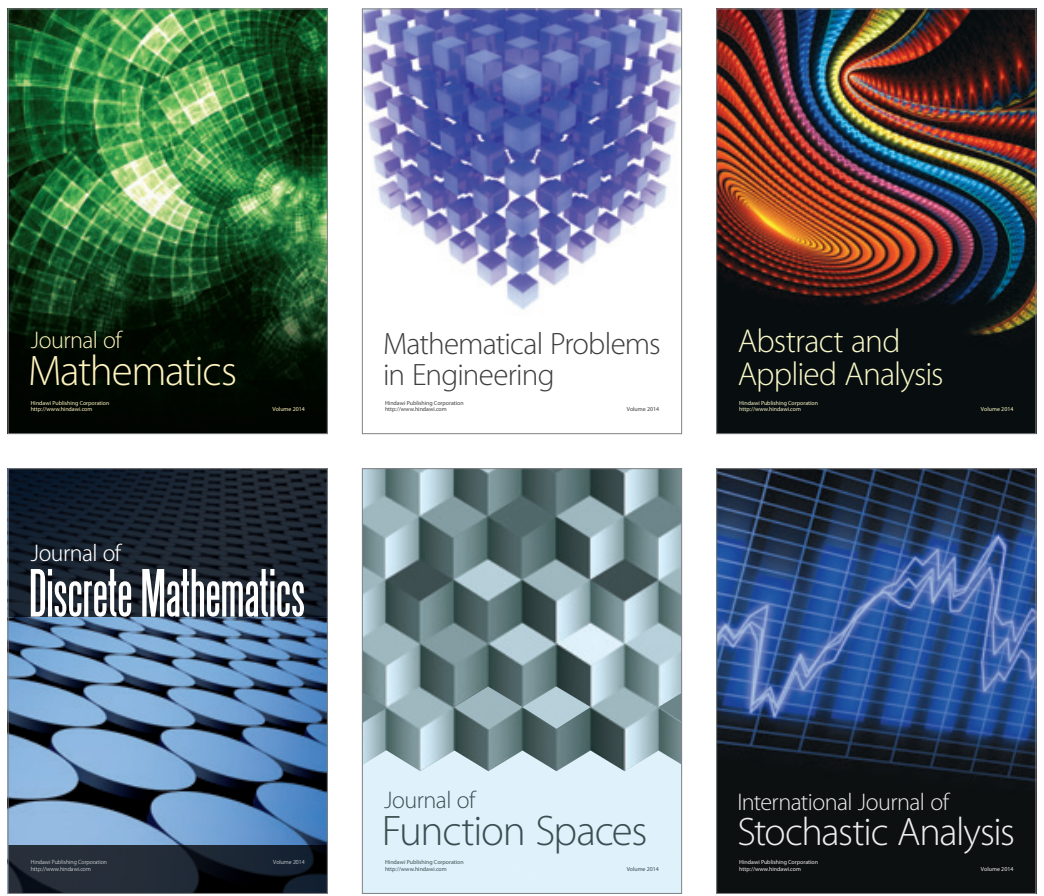

Journal of

Function Spaces

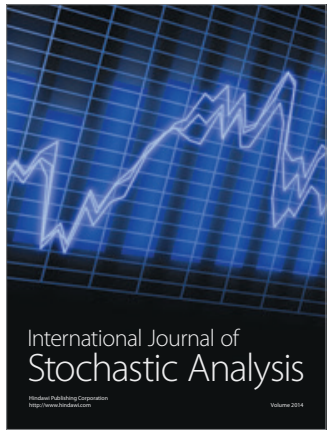

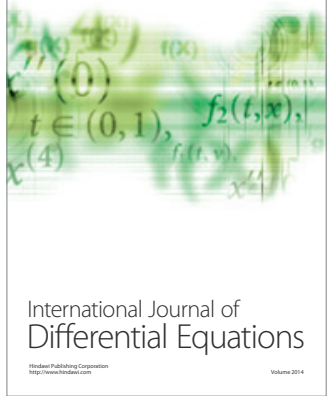
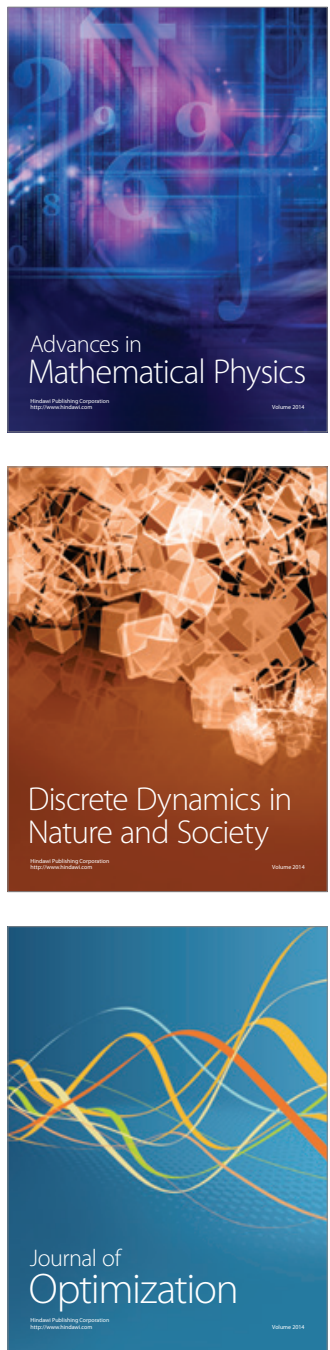